\title{
MEFM For Exact Solutions Of The (3+1) Dimensional KZK Equation and (3+1) Dimensional JM Equation
}

\author{
Seyma TULUCE DEMIRAY ${ }^{1 *}$, Sevgi KASTAL ${ }^{2}$ \\ ${ }^{1}$ Department of Mathematics, Faculty of Science and Letters, Osmaniye Korkut Ata University, Osmaniye, Turkey. \\ ${ }^{2}$ Department of Mathematics, Faculty of Science and Letters, Osmaniye Korkut Ata University, Osmaniye, Turkey.
}

Corresponding author ${ }^{*}$ e-posta1 : seymatuluce@gmail.com ORCID ID: http://orcid.org/0000-0002-8027-7290 e-posta²: kastalsevgi@gmail.com ORCIDID: http://orcid.org/0000-0001-9152-3023

Geliş Tarihi: 07.01.2021 Kabul Tarihi: 20.02.2021

\author{
Keywords \\ The $(3+1)$ dimensional \\ KZK Equation; (3+1)- \\ Dimensional Jimbo- \\ Miwa Equation; MEFM; \\ Dark Soliton Solution; \\ Trigonometric Function \\ Solutions; Hyperbolic \\ Solutions.
}

\begin{abstract}
In this study, we have applied the modified $\exp (-\Omega(\xi))$-expansion function method (MEFM) to obtain the exact travelling wave solutions for the $(3+1)$ dimensional Khokhlov-Zabolotskaya-Kuznetsov (KZK) equation and (3+1)-dimensional Jimbo-Miwa (JM) equation. Dark soliton solution, dark-bright soliton solution, hyperbolic function solution and trigonometric function solution of the $(3+1)$ dimensional KZK equation and (3+1)-dimensional JM equation have been found by using this method. After that, we have scratched the 2D and 3D graphs for all exact solutions obtained in this study by using Wolfram Mathematica 9. Thus, the graphical simulations openly show force of this method.
\end{abstract}

\section{$(3+1)$ Boyutlu KZK Denklemi ve (3+1) Boyutlu JM Denkleminin Tam Çözümleri için MEFM}

\author{
Anahtar kelimeler \\ $(3+1)$ boyutlu KZK \\ Denklemi; $(3+1)$ - \\ Boyutlu Jimbo-Miwa \\ Denklemi; MEFM; Dark \\ Soliton Çözümü; \\ Trigonometrik \\ Fonksiyon Çözümleri; \\ Hiperbolik Çözümler.
}

\begin{abstract}
Öz
Bu çalışmada, (3+1) boyutlu Khokhlov - Zabolotskaya - Kuznetsov (KZK) denklemi ve (3+1) boyutlu Jimbo-Miwa (JM) denklemlerinin yürüyen tam dalga çözümlerini elde etmek için, modifiye edilmiş exp $(-\Omega(\xi))$-açılım fonksiyon metodunu (MEFM) uyguladık. Bu yöntemle $(3+1)$ boyutlu KZK denkleminin ve $(3+1)$ boyutlu JM denkleminin dark soliton çözümü, dark-bright soliton çözümü, hiperbolik fonksiyon çözümü ve trigonometrik fonksiyon çözümü bulunmuştur. Daha sonra, bu çalışmada elde edilen tüm kesin çözümler için Wolfram Mathematica 9'u kullanarak 2 boyutlu ve 3 boyutlu grafikleri çizdik. Böylece, grafiksel gösterimler bu yöntemin gücünü açıkça göstermektedir.
\end{abstract}

(C) Afyon Kocatepe Üniversitesi

\section{Introduction}

In recent years nonlinear evolution equations (NLEEs) have become exclusive type of the branch of partial differential equations (PDEs). NLEEs are frequently used to expound a lot of physical events in the fields such as chemical kinematics, thermodynamic, fluid mechanic, optical fibers, heat transfer. Therefore, most of methods are established and applied for these problems. Some of these methods are Sine-Gordon expansion method (Ali et al. 2020), Hirota bilinear method (Ismael et al.
2020), Bifurcation method (Zheng et al. 2021), The modified auxiliary equation method (Akbar et al. 2020), Generalized Kudryashov method (Tuluce Demiray 2020), Simple Hirota's method (Ismael and Bulut 2020), ( $\left.m+1 / G^{\prime}\right)$-expansion method (Durur et al. 2020), finite difference method (Yokus and Kaya 2020), Sinh-Gordon function method (Yokus et al. 2020), (1/G')-expansion method (Yokus et al. 2020). The aim of this article, MEFM (Tuluce Demiray 2020, Tuluce Demiray and Bulut 2018) will be used to obtain new exact solutions (3+1) dimensional KZK equation and ( $3+1)$-dimensional JM equation. 
We consider the $(3+1)$ dimensional KZK equation (Akcagil and Aydemir 2016)

$s_{x t}+s_{x}^{2}+s s_{x x}+r s_{x x x}+n s_{y y}+m s_{z z}=0$,

where $m, n, r$ is real constants and $r \neq 0$.

Then, we investigate the (3+1)-dimensional JM equation (Zhang et al. 2009, Zayed et al. 2013)

$s_{x x x y}+6 s_{x} s_{y}+3 s v_{x x}+3 s_{x x} v+3 s_{y t}-3 s_{z z}=0$,

$s_{y}=v_{x}$.

Eq. (1) was natively created as a device for the defination of nonlinear acoustic beams (RozanovaPierrat 2006).

Our target is to get new exact solutions of $(3+1)$ dimensional KZK equation and (3+1)-dimensional JM equation by using suggested method. In Sec. 2, we clarify the technique. In Sec. 3, we apply suggested method to $(3+1)$ dimensional KZK equation and (3+1)-dimensional JM equation.

\section{Explanation of the Method}

For a known nonlinear partial differential equation is given as follows:

$K\left(s, s_{t}, s_{x}, s_{y}, s_{t t}, s_{x x}, s_{y y}, \cdots\right)=0$,

where $s=s(x, y, z, t)$ is an obscure function.

Step 1: Getting the transformation as

$s(x, y, t)=S(\sigma), \sigma=x+y+z-c t$,

Eq. (2) is turned into the following nonlinear equation:

$$
L\left(S, S^{\prime}, S^{\prime \prime}, S^{\prime \prime \prime}, \cdots\right)=0 .
$$

Step 2: Taking the following equation for Eq. (5) as solution:

$$
\begin{aligned}
& S(\sigma)=\frac{\sum_{i=0}^{p} A_{i}[\exp (-\vartheta(\sigma))]^{i}}{\sum_{j=0}^{q} B_{j}[\exp (-\vartheta(\sigma))]^{j}} \\
& =\frac{A_{0}+A_{1} \exp (-\vartheta)+\cdots+A_{p} \exp (p(-\vartheta))}{B_{0}+B_{1} \exp (-\vartheta)+\cdots+B_{q} \exp (q(-\vartheta))}
\end{aligned}
$$

where $A_{i}, B_{j},(0 \leq i \leq p, 0 \leq j \leq q)$ are constants, such that $A_{p} \neq 0, B_{q} \neq 0$. Also, $\vartheta=\vartheta(\sigma)$ is described as;

$\vartheta^{\prime}(\sigma)=\exp (-\vartheta(\sigma))+a \exp (\vartheta(\sigma))+b$.

Eq. (7) has the following solution families:

Family1: For $a \neq 0, b^{2}-4 a>0$,

$\vartheta(\sigma)=\ln \left(\frac{-\sqrt{b^{2}-4 a}}{2 a} \tanh \left(\frac{\sqrt{b^{2}-4 a}}{2}(\sigma+E)\right)-\frac{b}{2 a}\right)$.

Family2: When $a \neq 0, b^{2}-4 a<0$,

$\vartheta(\sigma)=\ln \left(\frac{\sqrt{-b^{2}+4 a}}{2 a} \tanh \left(\frac{\sqrt{-b^{2}+4 a}}{2}(\sigma+E)\right)-\frac{b}{2 a}\right)$.

Family3: When $a=0, b \neq 0$ and $b^{2}-4 a>0$,

$$
\vartheta(\sigma)=-\ln \left(\frac{b}{\exp (b(\sigma+E))-1}\right) .
$$

Family4: When $a \neq 0, b \neq 0$ and $b^{2}-4 a=0$,

$$
\vartheta(\sigma)=\ln \left(-\frac{2 b(\sigma+E)+4}{b^{2}(\sigma+E)}\right) .
$$


Family5: When $a=0, b=0$ and $b^{2}-4 a=0$,

$$
\vartheta(\sigma)=\ln (\sigma+E) \text {. }
$$

where $A_{i}, B_{j},(0 \leq i \leq p, 0 \leq j \leq q), E, b, a$ are constants to be obtained later.

Step 3: Setting Eq. (6) and Eq. (7) into Eq. (5), a system of $\mathrm{e}^{-\vartheta(\sigma)}$ can be obtained. We solve this system by using Wolfram Mathematica 9 to identify the coefficients $A_{i}, B_{j},(0 \leq i \leq p, 0 \leq j \leq q), E, b$ , a.

\section{Application to (3+1) dimensional KZK equation}

Getting the transformation as

$s(x, y, t)=S(\sigma), \quad \sigma=x+y+z-c t$,

Eq. (1) demeans

$$
(m+n-c) s+\frac{1}{2} s^{2}+r s^{\prime}=0 .
$$

By use of balance principle in Eq. (14), we get

$$
p=q+1 \text {. }
$$

If we get $q=1$ so $p=2$, we have

$$
S=\frac{A_{0}+A_{1} \exp (-\vartheta)+A_{2} \exp (2(-\vartheta))}{B_{0}+B_{1} \exp (-\vartheta)}=\frac{\Upsilon}{\Psi},
$$

and

$$
S^{\prime}=\frac{\Upsilon^{\prime} \Psi-\Psi^{\prime} \Upsilon}{\Psi^{2}}
$$

$$
S^{\prime \prime}=\frac{\Upsilon^{\prime \prime} \Psi^{3}-\Psi^{2} \Upsilon^{\prime} \Psi^{\prime}-\left(\Psi^{\prime \prime} \Upsilon+\Psi^{\prime} \Upsilon^{\prime}\right) \Psi^{2}+2\left(\Psi^{\prime}\right)^{2} \Upsilon \Psi}{\Psi^{4}},
$$

Thus, a system of $\mathrm{e}^{-\vartheta(\sigma)}$ can be obtained. We solve this system by using Wolfram Mathematica 9 to identify the coefficients $A_{i}, B_{j},(0 \leq i \leq p$, $0 \leq j \leq q), E, b, a$.

\section{Case 1:}

$A_{0}=\frac{i \sqrt{a} \sqrt{A_{1}^{3}}}{2 \sqrt{A_{1}-8 r B_{0}}}, A_{2}=-\frac{i \sqrt{A_{1}} \sqrt{A_{1}-8 r B_{0}}}{2 \sqrt{a}}$,

$B_{1}=-\frac{i \sqrt{A_{1}} \sqrt{A_{1}-8 r B_{0}}}{4 r \sqrt{a}}$,

$\lambda=\frac{8 i r \sqrt{a} B_{0}}{\sqrt{A_{1}} \sqrt{A_{1}-8 r B_{0}}}, c=m+n+\frac{4 i r \sqrt{a}\left(A_{1}-4 r B_{0}\right)}{\sqrt{A_{1}} \sqrt{A_{1}-8 r B_{0}}}$.

According to Eq. (19), we find trigonometric function solution for Eq. (1) as follows:

$s_{1}(x, y, z, t)=\frac{\left(2 r \sqrt{a} A_{1}^{3 / 2} K(-i+\tan [g(x, y, z, t)])^{2}\right)}{\left(P(D-K \tan [g(x, y, z, t)])\left(A_{1}+D(i+\tan [g(x, y, z, t)])\right)\right)},(20)$

where

$$
\begin{aligned}
& g(x, y, z, t)=\frac{\sqrt{a}\left(A_{1}-4 r B_{0}\right)\left(E+x+y+z-t\left(m+n+\frac{4 i r \sqrt{a}\left(A_{1}-4 r B_{0}\right)}{\sqrt{A_{1}} \sqrt{A_{1}-8 r B_{0}}}\right)\right)}{\sqrt{A_{1}} \sqrt{A_{1}-8 r B_{0}}} \\
& K=\left(A_{1}-4 r B_{0}\right), P=\sqrt{A_{1}-8 r B_{0}} \text { and } D=4 i r B_{0} .
\end{aligned}
$$

\section{Case 2:}

$A_{0}=\frac{4 i r \sqrt{a} B_{0}\left(A_{1}-2 r B_{0}\right)}{\sqrt{A_{1}} \sqrt{A_{1}-8 r B_{0}}}, A_{2}=-\frac{i \sqrt{A_{1}} \sqrt{A_{1}-8 r B_{0}}}{2 \sqrt{a}}$,

$$
B_{1}=-\frac{i \sqrt{A_{1}} \sqrt{A_{1}-8 r B_{0}}}{4 r \sqrt{a}},
$$

$$
\begin{aligned}
& b=\frac{i \sqrt{a}\left(3 A_{1}^{2}-8 r A_{1} B_{0}+16 r^{2} B_{0}^{2}\right)}{2 \sqrt{A_{1}} \sqrt{A_{1}-8 r B_{0}}\left(A_{1}-2 r B_{0}\right)}, \\
& c=m+n+\frac{i r \sqrt{a}\left(A_{1}-4 r B_{0}\right)\left(5 A_{1}-4 r B_{0}\right)}{\sqrt{A_{1}} \sqrt{A_{1}-8 r B_{0}}\left(A_{1}-2 r B_{0}\right)} .
\end{aligned}
$$


According to Eq. (21), we find trigonometric function solution for Eq. (1) as follows:

$s_{2}(x, y, z, t)=\frac{4 i r \sqrt{a}\left(A_{1}-2 r B_{0}+\frac{2 A_{1}\left(A_{1}-8 r B_{0}\right)\left(A_{1}-2 r B_{0}\right)}{P+i M \tan [\tau(x, y, z, t)]}\right)}{\sqrt{A_{1}} \sqrt{A_{1}-8 r B_{0}}}$,

where $M=\left(A_{1}-4 r B_{0}\right)\left(5 A_{1}-4 r B_{0}\right)$,

$P=3 A_{1}^{2}-8 r A_{1} B_{0}+16 r^{2} B_{0}^{2}$ and

$\tau(x, y, z, t)=\frac{\sqrt{a} M\left(E+x+y+z-t\left(m+n+\frac{i r \sqrt{a}\left(A_{1}-4 r B_{0}\right)\left(5 A_{1}-4 r B_{0}\right)}{2 \sqrt{A_{1}} \sqrt{A_{1}-8 r B_{0}}\left(A_{1}-2 r B_{0}\right)}\right)\right)}{\sqrt{A_{1}} \sqrt{A_{1}-8 r B_{0}}}$.

Case 3:

$A_{0}=\frac{B_{0}\left(A_{1}-2 r B_{0}\right)}{B_{1}}, A_{2}=2 r B_{1}$

$c=m+n-r b+\frac{A_{1}-2 r B_{0}}{B_{1}}$,

$a=-\frac{\left(A_{1}-2 r B_{0}\right)\left(A_{1}-2 r\left(B_{0}+b B_{1}\right)\right)}{4 r^{2} B_{1}^{2}}$.

According to Eq. (23), we get dark optical soliton solution for Eq. (1) as follows:

$s_{3}(x, y, z, t)=\frac{\left(A_{1}-2 r B_{0}\right)\left(B_{1}+\frac{A_{1}-2 r\left(B_{0}+b B_{1}\right)}{A_{1}-r\left(2 B_{0}+b B_{1}\right) \tanh [k(x, y, x, t)]}\right)}{B_{1}^{2}},(24)$

where

$k(x, y, z, t)=\frac{\left(E+x+y+z-t\left(m+n-r b+\frac{A_{1}-2 r B_{0}}{B_{1}}\right)\right)\left(A_{1}-r\left(2 B_{0}+b B_{1}\right)\right)}{2 r B_{1}}$.

\section{Application to (3+1) dimensional JM Equation}

Getting the transformation as

$s=s(\xi), \xi=k x+m y+n z+w t$,
Eq. (2) demeans

$k^{3} m s^{\prime \prime}+3 k m s^{2}+\left(3 w m-3 n^{2}\right) s=0$.

By use of balance principle in Eq. (26), one can procured

$$
p=q+2 \text {. }
$$

If we get $q=1$ so $p=3$, we have

$S=\frac{A_{0}+A_{1} \exp (-\vartheta)+A_{2} \exp (2(-\vartheta))+A_{3} \exp (3(-\vartheta))}{B_{0}+B_{1} \exp (-\vartheta)+B_{2} \exp (-\vartheta)}=\frac{\Upsilon}{\Psi}$,

and

$S^{\prime}=\frac{\Upsilon^{\prime} \Psi-\Psi^{\prime} \Upsilon}{\Psi^{2}}$

$S^{\prime \prime}=\frac{\Upsilon^{\prime \prime} \Psi^{3}-\Psi^{2} \Upsilon^{\prime} \Psi^{\prime}-\left(\Psi^{\prime \prime} \Upsilon+\Psi^{\prime} \Upsilon^{\prime}\right) \Psi^{2}+2\left(\Psi^{\prime}\right)^{2} \Upsilon \Psi}{\Psi^{4}}$.

Thus, a system of $\mathrm{e}^{-\vartheta(\sigma)}$ can be obtained. We solve this system by using Wolfram Mathematica 9 to identify the coefficients $A_{i}, B_{j},(0 \leq i \leq p$, $0 \leq j \leq q), E, b, a$.

\section{Case 1:}

$A_{0}=-2 k^{2} a B_{0}, A_{1}=\frac{A_{2} B_{0}+2 k^{2}\left(B_{0}^{2}-a B_{1}^{2}\right)}{B_{1}}$,

$A_{3}=-2 k^{2} B_{1}, \lambda=-\frac{A_{2}+2 k^{2} B_{0}}{2 k^{2} B_{1}}$,

$w=\frac{n^{2}}{m}+\frac{4 k^{3} a}{3}-\frac{\left(A_{2}+2 k^{2} B_{0}\right)^{2}}{12 k^{2} B_{1}^{2}}$.

According to Eq. (31), we obtain dark-bright optical soliton solution for Eq. (2) as follows: 
$s_{1}(x, y, z, t)=\frac{2 k^{2} a \operatorname{sech}[r(x, y, z, t)]^{2}\left(\left(A_{2}+2 k^{2} B_{0}\right)^{2}-16 k^{4} a B_{1}^{2}\right)}{\left(A_{2}+k^{2}\left(2 B_{0}-2 \sqrt{-4 a+\frac{\left(A_{2}+2 k^{2} B_{0}\right)^{2}}{4 k^{4} B_{1}^{2}}} B_{1} \tanh [r(x, y, z, t)]\right)\right)^{2}},(32)$

where

$r(x, y, z, t)=\frac{1}{2}\left(E+k x+m y+n z+t\left(\frac{m^{2}}{n}+\frac{4 k^{3} a}{3}-\frac{\left(A_{2}+2 k^{2} B_{0}\right)^{2}}{12 k B_{1}^{2}}\right)\right) M$

and

$M=\sqrt{-4 a+\frac{\left(A_{2}+2 k^{2} B_{0}\right)^{2}}{4 k^{4} B_{1}^{2}}}$.

Case 2:

$A_{0}=-2 k^{2} a B_{0}, A_{1}=a A_{3}-2 k^{2} b B_{0}$,

$A_{2}=b A_{3}-2 k^{2} B_{0}$

$B_{1}=-\frac{A_{3}}{2 k^{2}}, w=\frac{n^{2}}{m}-\frac{1}{3} k^{3}\left(b^{2}-4 a\right)$.

According to Eq. (33), we find hyperbolic function solution for Eq. (2) as follows:

$s_{2}(x, y, z, t)=\frac{2 k^{2}\left(b^{2}-4 a\right) a}{\left(b \cosh [h(x, y, z, t)]+\sqrt{b^{2}-4 a} \sinh [h(x, y, z, t)]\right)^{2}},(34)$

where

$h(x, y, z, t)=\frac{1}{2}\left(E+\frac{n^{2} t}{m}+k x+m y+n z-\frac{1}{3} k^{3} t\left(b^{2}-4 a\right)\right) \sqrt{b^{2}-4 a}$.

\section{Case 3:}

$$
\begin{aligned}
& A_{0}=\frac{a A_{3} B_{0}}{B_{1}}, A_{1}=A_{3}\left(a+\frac{b B_{0}}{B_{1}}\right), \\
& A_{2}=A_{3}\left(b+\frac{B_{0}}{B_{1}}\right), k=-\frac{i \sqrt{A_{3}}}{\sqrt{2 B_{1}}},
\end{aligned}
$$

$$
w=\frac{-i \sqrt{2} m\left(b^{2}-4 a\right) \sqrt{A_{3}^{3}}+12 n^{2} \sqrt{B_{1}^{3}}}{12 m \sqrt{B_{1}^{3}}} .
$$

According to Eq. (35), we obtain hyperbolic function solution for Eq. (2) as follows:

$$
s_{3}(x, y, z, t)=\frac{a\left(-b^{2}+4 a\right) A_{3}}{\left(b \cosh [f(x, y, z, t)]+\sqrt{b^{2}-4 a} \sinh [f(x, y, z, t)]\right)^{2} B_{1}},
$$

where

$f(x, y, z, t)=\frac{\sqrt{P}\left(-i \sqrt{2} m t P \sqrt{A_{3}^{3}}-6 i \sqrt{2 A_{3}} m x B_{1}+12\left(n^{2} t+m(E+m y+n z)\right) \sqrt{\left.B_{1}^{3}\right)}\right.}{24 m \sqrt{B_{1}^{3}}}$

and $P=\left(b^{2}-4 a\right)$.

\section{Case 4:}

$A_{0}=\frac{B_{0}\left(-8 k^{4} a-\frac{\left(A_{2}+2 k^{2} B_{0}\right)^{2}}{B_{1}^{2}}\right)}{12 k^{2}}$,
$A_{1}=-\frac{A_{2}^{2}-8 k^{2} A_{2} B_{0}+4 k^{4}\left(-5 B_{0}^{2}+2 a B_{1}^{2}\right)}{12 k^{2} B_{1}}$,

$A_{3}=-2 k^{2} B_{1}, b=-\frac{A_{2}+2 k^{2} B_{0}}{2 k^{2} B_{1}}$,

$w=\frac{n^{2}}{m}-\frac{4 k^{3} a}{3}+\frac{\left(A_{2}+2 k^{2} B_{0}\right)^{2}}{k B_{1}^{2}}$.

According to Eq. (37), we find dark optical soliton solution for Eq. (2) as follows:

$$
s_{4}(x, y, z, t)=\left(\begin{array}{l}
\frac{\left(4 k^{2} a^{2}\left(-24 k^{4} B_{1}^{2}-\frac{M(L+K \tanh [h(x, y, z, t)])^{2}}{4 a^{2}}\right)\right)}{\left(3\left(A_{2}+k^{2}\left(2 B_{0}-2 K \tanh [h(x, y, z, t)]\right)^{2}\right)\right.} \\
\left.+\frac{\left(4 k^{2} a^{2}\left(\frac{N\left(A_{2}+k^{2}\left(2 B_{0}-2 K B_{1} \tanh [h(x, y, z, t)]\right)\right)}{a}\right)\right)}{\left(3\left(A_{2}+k^{2}\left(2 B_{0}-2 K \tanh [h(x, y, z, t)]\right)\right)^{2}\right)}\right),
\end{array}\right)
$$


$h(x, y, z, t)=\frac{1}{2} K\left(E+\frac{q^{2} t}{p}+k x+m y+n z-\frac{4}{3} k^{3} t a+\frac{t\left(A_{2}+2 k^{2} B_{0}\right)^{2}}{12 k B_{1}^{2}}\right)$

and

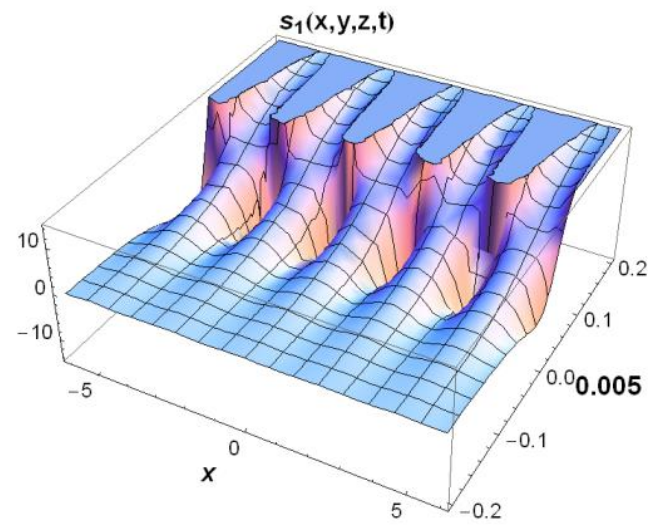

$$
\begin{aligned}
& K=\sqrt{-4 a+\frac{\left(A_{2}+2 k^{2} B_{0}\right)^{2}}{4 k^{4} B_{1}^{2}}}, \\
& M=\left(\left(A_{2}+2 k^{2} B_{0}\right)^{2}+8 k^{4} a B_{1}^{2}\right), \\
& N=3\left(A_{2}+2 k^{2} B_{0}\right), L=-\frac{A_{2}+2 k^{2} B_{0}}{2 k^{2} B_{1}} .
\end{aligned}
$$

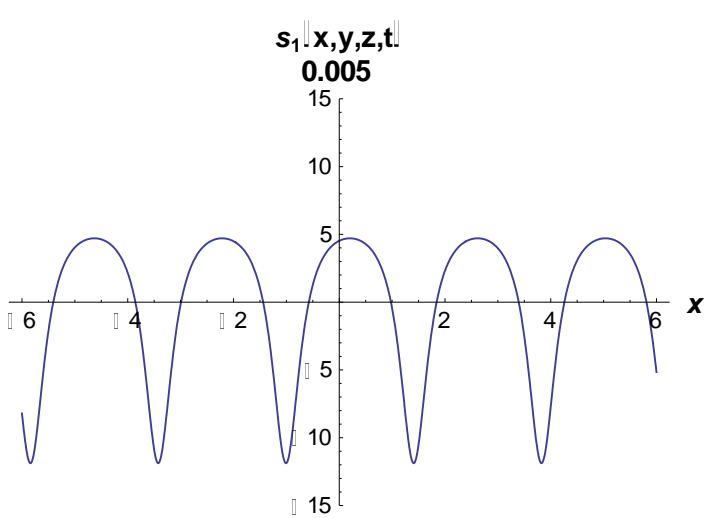

Fig.1. Three-dimensional and two-dimensional plots of imaginary values of Eq. (20) for $y=3, z=-5, m=1, n=1$, $r=3, E=0.6, A_{1}=4, B_{0}=-0.1, a=0.4,-6<x<6,-0.2<t<0.2$ and $t=0.005$ for 2D plot.
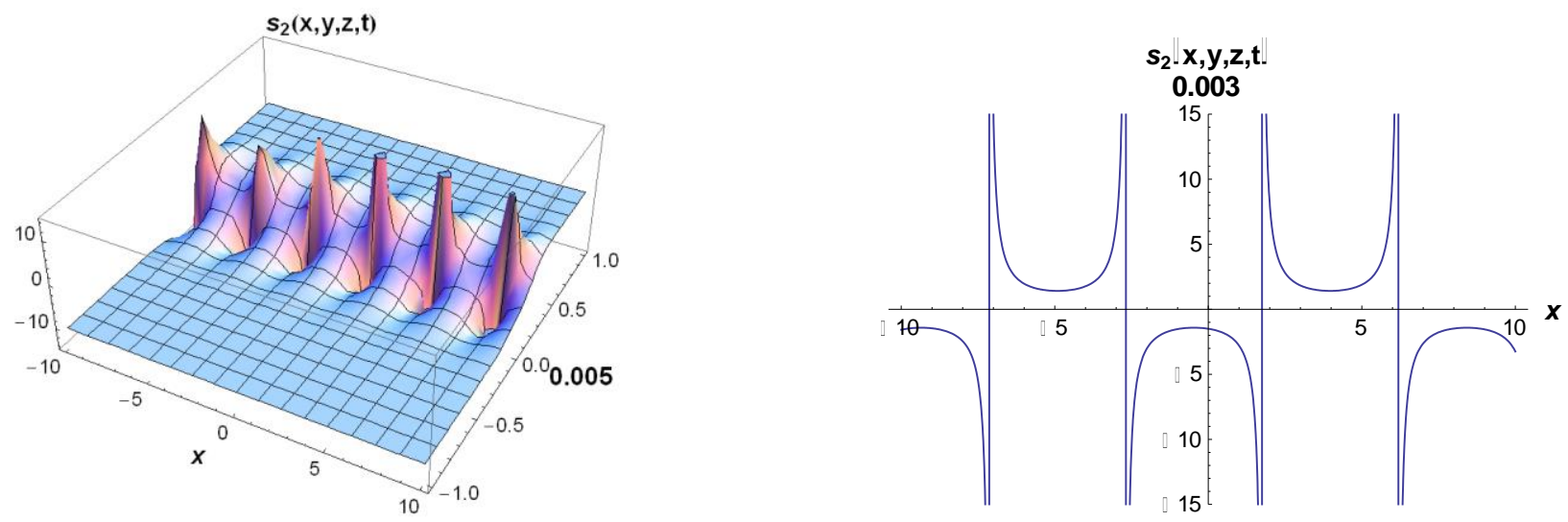

Fig.2. Three-dimensional and two-dimensional plots of imaginary values of Eq. (22) for $a=0.4, y=5, z=-6, r=-3$ , $A_{1}=20, E=0.05,-10<x<10,-1<t<1$ and $t=0.005$ for $2 \mathrm{D}$ plot. 

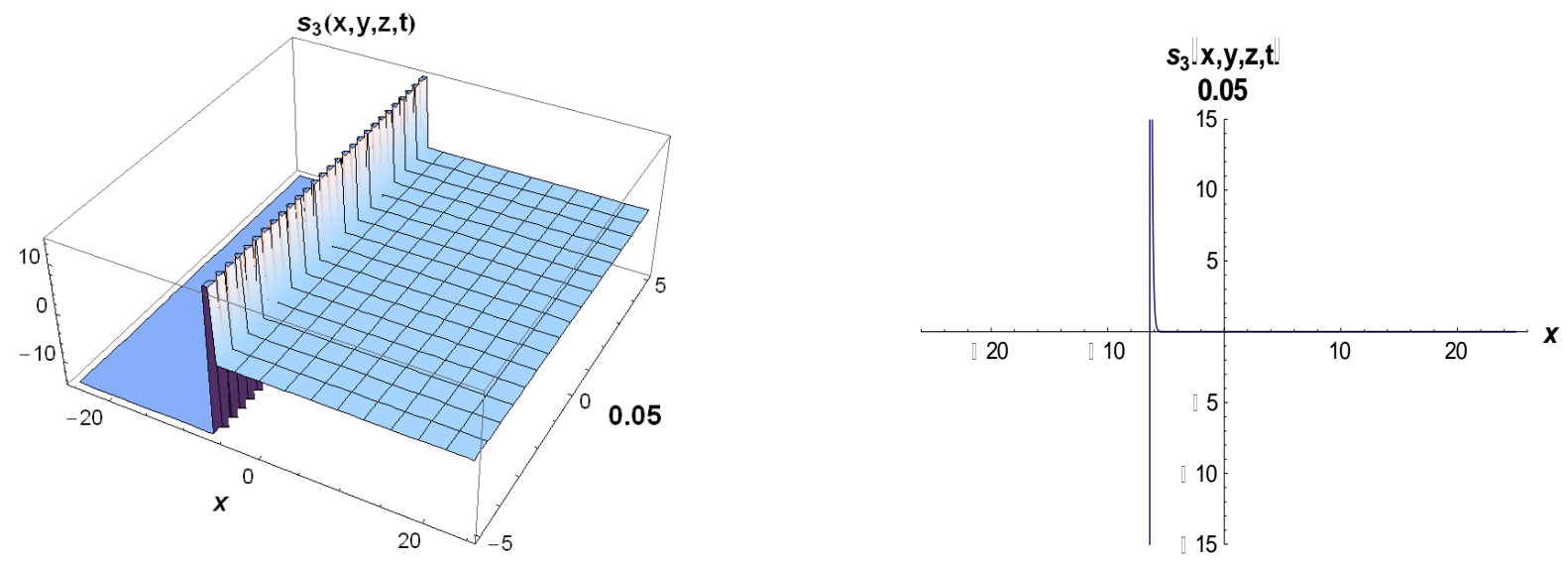

Fig.3. Three-dimensional and two-dimensional plots of real values of Eq. (24) for $b=5, y=3, z=1.2, m=3, n=2$, $r=5, A_{1}=4, B_{0}=-0.3, B_{1}=-0.3, E=-0.1,-25<x<25,-5<t<5$ and $t=0.005$ for $2 \mathrm{D}$ plot.
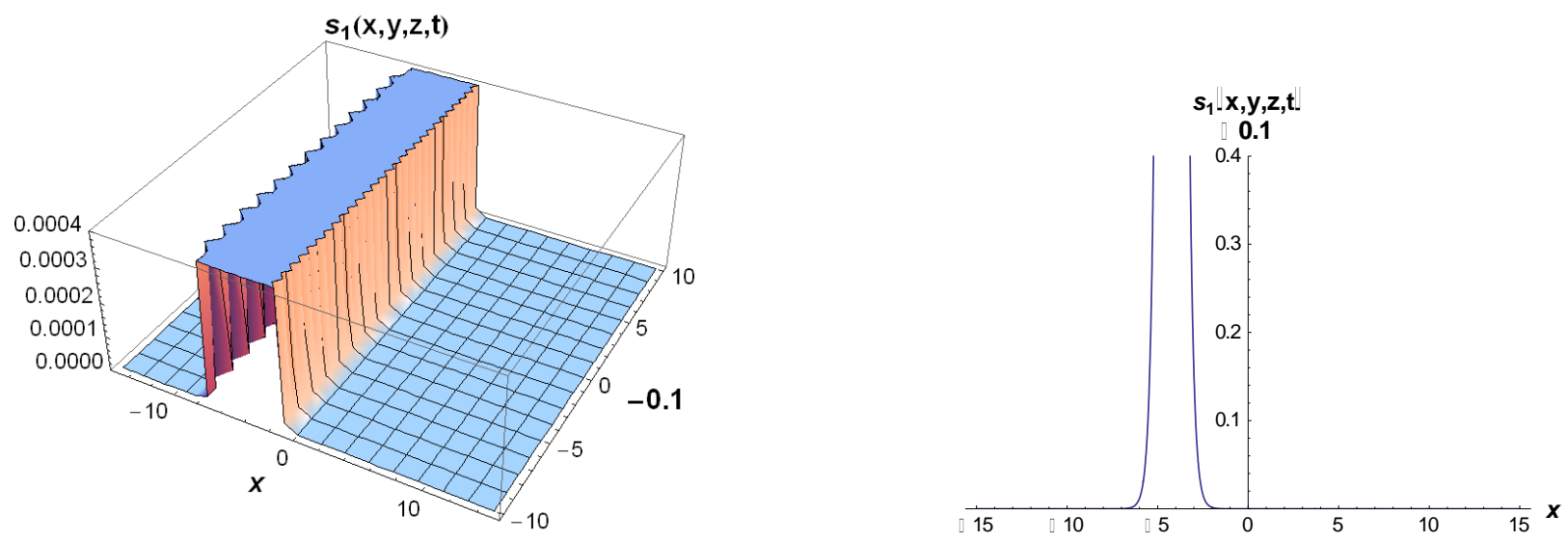

Fig.4. Three-dimensional and two-dimensional plots of Eq. (32) for $A_{2}=-2, B_{0}=-3, B_{1}=1.2, E=-0.2, k=0.2$, $y=-1, z=1, m=0.5, a=5, n=3,-25<x<25,-10<t<10$ and $t=-0.1$ for 2D plot.
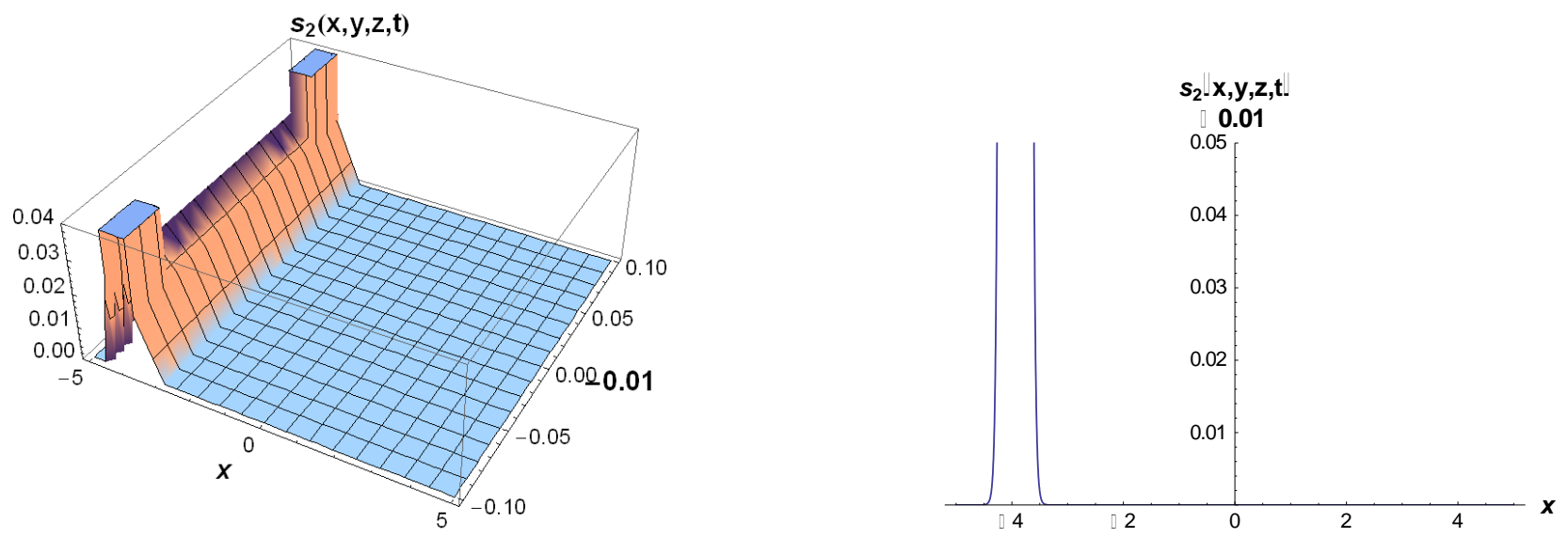

Fig.5. Three-dimensional and two-dimensional plots of Eq. (34) for $y=-2, z=0.5, m=-4, n=-1.2, E=-5.3$, $k=5, a=2, b=7,-5<x<5,-0.1<t<0.1$ and $t=-0.01$ for $2 \mathrm{D}$ plot. 

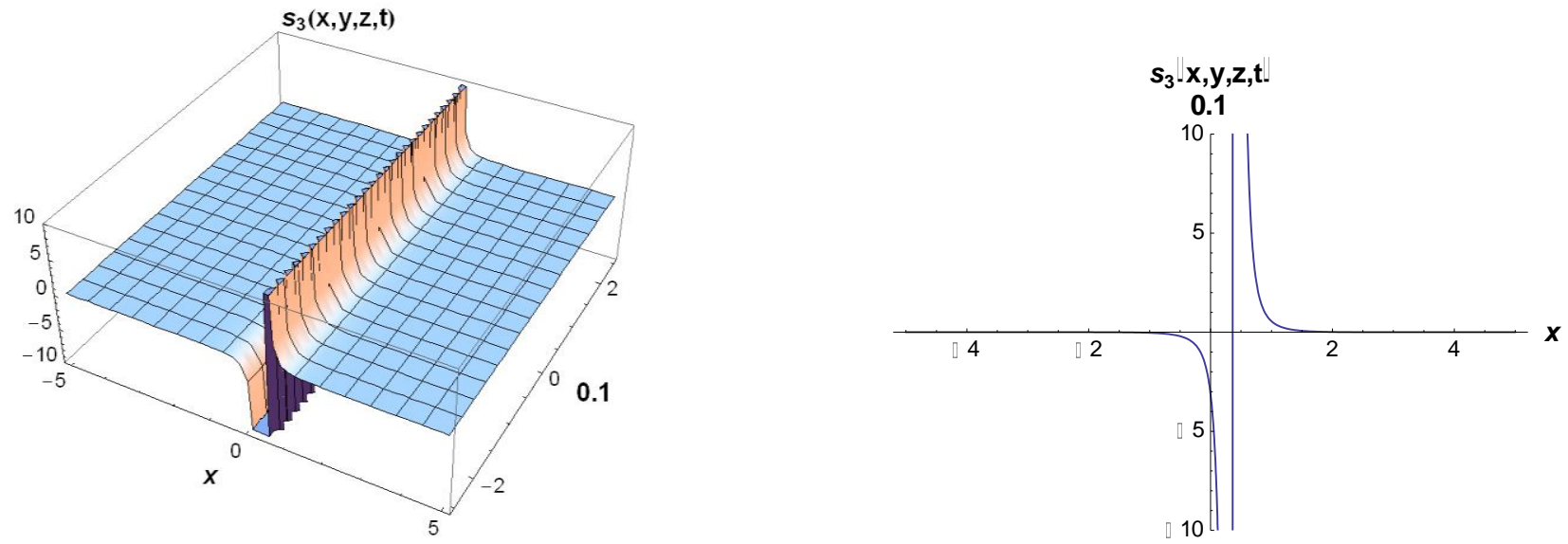

Fig.6. Three-dimensional and two-dimensional plots of Eq. (36) for $y=0.3, z=-0.002, m=-3.5, n=20.2$, $E=-0.1, a=3, b=2,-5<x<5,-0.1<t<0.1$ and $t=-0.01$ for $2 \mathrm{D}$ plot.
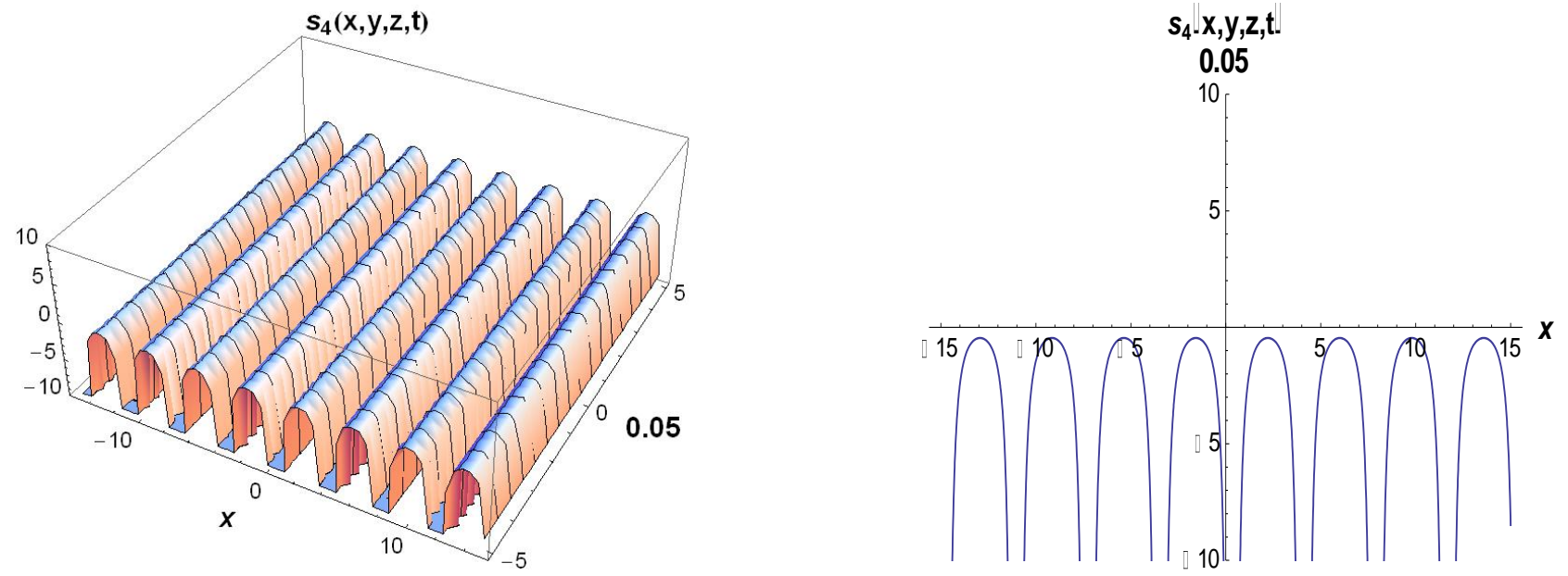

Fig.7. Three-dimensional and two-dimensional plots of Eq. (38) for $y=3.2, z=2, m=0.5, n=0.2, E=3.1, a=5$, $b=2,-15<x<15,-5<t<5$ and $t=0.05$ for $2 \mathrm{D}$ graph.

\section{Results and Discussion}

We obtain some exact solutions of $(3+1)$ dimensional KZK equation and (3+1)-dimensional $J M$ equation by applying MEFM. These solutions were controlled in Wolfram Mathematica 9. We prove their accuracy by graphically representing these obtained results by aid of Wolfram Mathematica 9. MEFM, which is easier to apply than other methods, is a very effective and reliable method for finding solutions to NLEEs.

\section{Conclusion}

In this paper, we make use of MEFM to seek solutions of $(3+1)$ dimensional KZK equation and $(3+1)$-dimensional JM equation. Then, we draw two and three dimensional graphs of dark optical soliton solutions, trigonometric function solution, dark- bright optical soliton solution and hyperbolic function solutions of these equations via Wolfram Mathematica 9.

As a result of these datas, it has been deduced that MEFM is extremely credible and strong in the sense that finding exact solutions. The paper shows that the MEFM algorithm is efficient and can be used for many other NLEEs in mathematical physics.

\section{REFERENCES}

Akbar, M. A., Mohd. Ali, N., Tanjim, T., 2020. Adequate soliton solutions to the perturbed Boussinesq equation and the KdV-Caudrey-Dodd-Gibbon Equation. Journal of King Saud University - Science, $32,6,2777-2785$.

Akcagil, S., Aydemir, T., 2016. New exact solutions for the Khokhlov-Zabolotskaya-Kuznetsov, the Newell- 
Whitehead-Segel and the Rabinovich wave equations by using a new modification of the tanh-coth method. Cogent Mathematics, 3, 1, 1193104, 1-12.

Ali, Khalid K., Osman, M.S., Mahmoud Abdel-Aty, 2020. New optical solitary wave solutions of Fokas-Lenells equation in optical fiber via Sine-Gordon expansion method. Alexandria Engineering Journal, 59, 3, 11911196.

Durur, H., Ilhan, E., Bulut, H., 2020. Novel Complex Wave Solutions of the (2+1)-Dimensional Hyperbolic Nonlinear Schrödinger Equation. Fractal and Fractional, 4, 41, 1-8.

Ismael, H. F., Bulut, H., 2020. Multi soliton solutions, Mlump waves and mixed solution lump solutions to the Sawada-Kotera equation in $(2+1)$ dimensions, Chinese Journal of Physics, In press, 19, 1-8.

Ismael, H. F., Bulut, H., Park, C., Osman, M.S., 2020. Mlump, N-soliton solutions, and the collision phenomena for the $(2+1)$-dimensional Date-JimboKashiwara-Miwa equation. Results in Physics, 19, 103329, 1-6.

Rozanova-Pierrat, A., 2006. Mathematical analysis of Khokhlov-Zabolotskaya-Kuznetsov (KZK) equation, hal-00112147.

Tuluce Demiray, S., 2020. New Solitions of Biswas-Arshed equation with beta time Derivative. Optik, 222, 165405, 1-5.

Tuluce Demiray, S., 2020. New Soliton Solitions of Optical Pulse Envolepe $E(Z, \tau)$ with Beta Time Derivative. Optik, 223, 165453, 1-6.

Tuluce Demiray, S., Bulut, H., 2018. A New Method for (4+1) Dimensional Fokas Equation, ITM Web of Conferences. 22, 01065, 1-5.

Yokus, A., Kaya, D., 2020. Comparison exact and numerical simulation of the traveling wave solution in nonlinear Dynamics. International Journal of Modern Physics B, 34, 29, 2050282, 1-22.

Yokus, A., Durur, H., Taher, A. N., Abu-Zinadah, H., Tuz, M., Ahmad, H., 2020. Study on the applications of two analytical methods for the construction of traveling wave solutions of the modified equal width equation. Open Physics, 18, 1, 1003-1010.

Yokus, A., Durur, H., Ahmad, H., Yao, S. W., 2020. Construction of Different Types Analytic Solutions for the Zhiber-Shabat Equation. Mathematics, 8, 908, 116.
Zayed, E. M. E., Amer, Y. A., Ahmed, H. A., 2013. Functional variable method and its applications for finding exact solutions of nonlinear PDEs in mathematical physics. Scientific Research and Essays, 8, 42, 2068-2074.

Zhang, S., Sun, Y.N., Ba, J.M., Dong, L., 2009. Explicit and exact solutions with multiple arbitrary analytic functions of Jimbo-Miwa Equation. Applications and Applied Mathematics, 4, 279-289.

Zheng, X., Xiao, Q., Ouyang, Z., 2021. A smooth soliton solution and a periodic cuspon solution of the Novikov equation. Applied Mathematics Letters, 112, 106786, 1-7. 